\title{
EFECTO DE LA HUMEDAD DE EQUILIBRIO EN LA RUGOSIDAD DE TABLEROS DE FIBRAS DE DENSIDAD MEDIA (MDF)
}

\section{EFFECT OF THE EQUILIBRIUM MOISTURE ON THE ROUGHNESS OF MEDIUM DENSITY FIBERBOARDS (MDF)}

Aldo Rolleri'; Edmone Roffael ${ }^{2}$

\begin{abstract}
RESUMEN
La rugosidad superficial es primariamente una función de las propiedades de la materia prima, pero otros factores como la cantidad y el tipo de resina, ciclo de prensado, lijado y contenido de humedad de los tableros pueden afectar la rugosidad y otras propiedades de la superficie. En este estudio los efectos del contenido de humedad de equilibro en superficies de tableros de densidad media (MDF), fueron evaluados usando diferentes materias primas en sus superficies como también diferentes adhesivos. El análisis estadístico confirma que la relación entre el contenido de humedad de equilibrio y la rugosidad media es una compleja función de interacciones entre muchas variables, pero donde tiene un papel relevante desde el punto de vista practico el tipo de adhesivos.
\end{abstract}

Palabras claves: rugosidad, humedad, MDF

\begin{abstract}
The surface roughness is primarily a function of the raw material properties, but other factors like type and amount of resin, press cycle, sanding and moisture content of the boards may also affect the roughness and other surface properties. In this study the effect of the equilibrium moisture content on medium density fiberboards (MDF) surfaces was evaluate using different raw materials in their surface layers as well as different binders. The statistical analysis confirmed that the relationship between equilibrium moisture content and average roughness are a complex function of interactions between many variables.
\end{abstract}

Key words: roughness, moisture, MDF

\section{INTRODUCCION}

El uso de paneles base fibra de madera es cada vez mas demandado por la uniformidad de sus propiedades físico-químicas si se le compara con aquellas encontradas en piezas de madera y es ampliamente percibido como un producto que a futuro remplace la madera aserrada (Roffael and Schäfer, 1997). Se suma a lo anterior el uso de tratamientos superficiales que tienen como finalidad brindar protección o ennoblecer productos base madera. El buen funcionamiento de estos tratamientos depende fuertemente de las propiedades que presenten las superficies de los substratos base madera utilizados

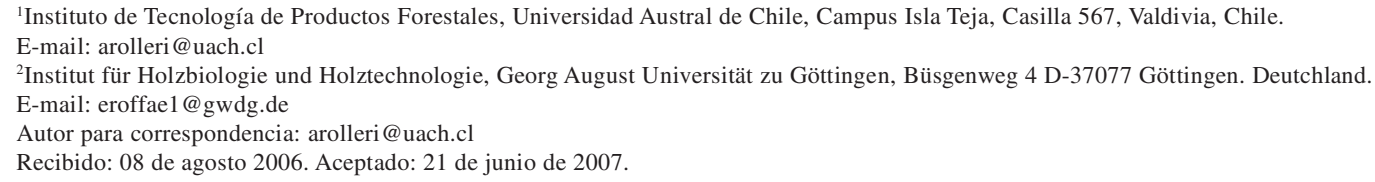


(Roffael, 1993). Las superficies de estos substratos pueden ser caracterizadas analizando la textura que ellas presentan, particularmente la rugosidad intrínseca en ella. La rugosidad define como se siente una superficie, su apariencia, como esta trabaja en contacto con otra superficie y como esta se comporta al ser recubierta o pintada (PDI, 1998). La caracterización de una superficie emplea parámetros estadísticos y matemáticos para su interpretación. El mas importante de todos es el llamado $\left(\mathrm{R}_{\mathrm{a}}\right)$ rugosidad media. Este es la distancia promedio entre el perfil superficial y la línea media (Hizoroglu, 1996). $\mathrm{R}_{\mathrm{a}}$ es también llamado desviación media aritmética y se define como la profundidad media de todas las profundidades entre el perfil superficial y la línea media. $\mathrm{R}_{\mathrm{a}}$ es también definida como la altura de un rectángulo de largo Lm y cuya área es igual aquella entre el perfil de la superficie y la línea media (Östman, 1983).

Bajo diferentes condiciones climáticas muchas características físicas y químicas de las superficies de los tableros base fibra de madera dependen de las especie y de los adhesivos utilizados. Muchas publicaciones tratan el comportamiento higroscópico de la madera presente en muchos productos, sin embargo, no muchas consideran la influencia de las condiciones climáticas en las propiedades de superficie de diferentes tableros base madera encolados con diferentes resinas.

El objetivo general de este estudio fue, evaluar la influencia del almacenamiento bajo tres condiciones climáticas $\left(20^{\circ} \mathrm{C} / 30 \%\right.$ humedad relativa, $20{ }^{\circ} \mathrm{C} / 65 \%$ humedad relativa y $20{ }^{\circ} \mathrm{C} / 85 \%$ humedad relativa) en la rugosidad de tableros de fibra de densidad media (MDF).

Los objetivos especificos fueron:

-efecto de fibras frescas, recicladas y partículas de corcho en la rugosidad de tableros base madera encolados con resinas melamina-urea- formaldehido (resinas-MUF)

-efecto de fibras frescas, recicladas y partículas de corcho en la rugosidad de tableros base madera encolados con resinas tanino-ureaformaldehido (resinas-TF).

\section{MATERIAL Y METODO}

Durante el presente trabajo tableros monocapa y tricapa fueron producidos usando diferentes resinas para encolar diferentes materias primas presentes en sus capas superficiales. Las materias primas fueron industrialmente producidas, fibras frescas provenientes de un proceso de pulpaje termo-mecánico TMP, fibras recicladas a apartir de tableros de fibras encolados con resinas UF y finalmente partículas de corcho utilizadas para recubrir capas superficiales de tableros cuyas capas centrales son de fibras.

Las fibras frescas fueron suministradas por una compañía alemana. Las pulpas fueron obtenidas a partir de una mezcla de Picea abies (ciprés) y Pinus sylvestris (pino). La temperatura de pulpaje fue aproximadamente de $180{ }^{\circ} \mathrm{C}$. Las fibras desfibradas fueron transportadas al Instituto de Biología y Tecnología de la Madera en la Georg-August Universität en Alemania y secadas en un horno a $70^{\circ} \mathrm{C}$ hasta una contenido de humedad cercano al 5\%. La misma compañia ofrece tableros de fibras de densidad media (MDF) para la producción de fibras recicladas; las fibras recicladas fueron producidas por un proceso termo-hidrolítico. El proceso fue el siguiente:

Los tableros MDF no recubiertos de $7 \mathrm{~mm}$ con resinas UF fueron cortados en piezas de $5.0 \mathrm{~cm} *$ $5.0 \mathrm{~cm}$, para luego ser destruidas por una máquina especial. Posteriormente el material $(<0.5 \mathrm{~mm})$ fue rechazado. El material MDF (6.0 Kg. secos) fue pulpado en un autoclave de 50 litros usando $1 \%$ de hidróxido de sodio $(\mathrm{NaOH})$ (basado en el material secado en horno) a una relación de licor de 1:6. El auto clave fue calentado hasta una temperatura máxima de $130^{\circ} \mathrm{C}$, después de alcanzada esta temperatura 
la cocción fue continuada por 1 hora. Durante el pulpaje el autoclave rotaba 360 grados. Después de la cocción las fibras fueron dejadas enfriar en el autoclave hasta temperatura ambiente alrededor de 18 horas. Las fibras obtenidas fueron secadas hasta un contenido de humedad del $5 \%$. Después de secadas las fibras fueron procesadas en un molino Pallmnnn-mill PXL a $12400 \mathrm{rpm}$ hasta su disgregación. Las fibras fueron tamizadas y las fibras finas y gruesas fueron separadas. Para producir MDF reciclado sólo fibras entre $0.2 \mathrm{~mm}$ y $2.0 \mathrm{~mm}$ fueron usadas para las capas superficiales. Las partículas de corcho fueron suministradas por una compañia alemana. Estas partículas de corcho utilizadas en capas superficiales fueron tambien tamizadas y clasificadas entre un rango de $0.2 \mathrm{~mm}$ y $1.0 \mathrm{~mm}$. Las partículas de corcho fueron secadas hasta aproximadamente un $2.0 \%$ de contenido de humedad.

Para la preparación de los tableros de fibras de densidad media (MDF) se utilizó resina comercial del tipo melamina-urea-formaldehido (resinas-MUF, BASF K407 con un contenido de melamina de alrededor del $1 \%$ ) y resina tanino-formaldehido (resinas-TF). Seis diferentes tipos de tableros MDF fueron hechos. De cada tipo se hicieron tres repeticiones obteniendo un total de 18 tableros. Estos tableros correspondieron a los del tipo monocapa con fibras frescas, monocapa con fibras recicladas y un último tricapa con capa central de fibras frescas y capas superficiales de partículas de corcho. La tabla 1. muestra las condiciones para la preparación de los diferentes MDF:

Tabla 1: Condiciones para la preparación de los diferentes MDF

\begin{tabular}{|c|c|c|c|c|c|c|}
\hline & $\overline{\text { V1 }}$ & $\overline{\mathrm{V} 2}$ & $\overline{\mathrm{V} 3}$ & V4 & V5 & $\overline{V 6}$ \\
\hline Tipo de adhesivo: & MUF-resinas & TF-resinas (1) & MUF-resinas & TF-resinas (1) & MUF-resinas & TF-resinas (1) \\
\hline Tipo de tanino: & & GT 5 (2) & & GT 5 (2) & & GT $5(2)$ \\
\hline Nivel de adhesivo: & $12 \%$ & 14 & $12 \%$ & 14 & $12 \%(3)$ & $10 \%(3)$ \\
\hline & & & & & $10 \%(4)$ & $14 \%(4)$ \\
\hline Formaldehído: & & 10,5 & & 10,5 & & $6.5 \%(5)$ \\
\hline & & & & & & $8.5 \%(6)$ \\
\hline Endurecedor & Sulf. de amonio & & Sulf. de amonio & & Sulf. de amonio & \\
\hline Nivel del endurecedor & $4 \%$ & & $4 \%$ & & $4 \%(8)$ & \\
\hline Tiempo de prensado: & $30 \mathrm{~s} / \mathrm{mm}$ & $40 \mathrm{~s} / \mathrm{mm}$ & $30 \mathrm{~s} / \mathrm{mm}$ & $40 \mathrm{~s} / \mathrm{mm}$ & $\begin{array}{c}4 \%(9) \\
60 \mathrm{~s} / \mathrm{mm}(7)\end{array}$ & $60 \mathrm{~s} / \mathrm{mm}(7)$ \\
\hline
\end{tabular}

(1) (45\% contenido de sólidos), (2) Colatan GT 5 Industria Argentina, (3) $10 \%$ (sólidos basados en fibras secas), (4) Capa superficial 14 $\%$ Sulfato de amonio (sólidos basados en resina seca (tanino)), (5) Capa superficial $6.5 \%$ Sulfato de amonio (sólidos basados en resina seca (tanino)), (6) Capa media $8.5 \%$ Sulfato de amonio (sólidos basados en resina seca (tanino)), (7) $60 \mathrm{~s} / \mathrm{mm}$ (excl. tiempo de cierre de la prensa), (8) Capa superficial 4,0\% Sulfato de amonio (sólidos basados en resina seca MUF), (9) Capa media 4,0 \% Sulfato de amonio (sólidos basados en resina seca MUF )

Después del prensado y el enfriamiento los tableros de densidad media fueron trimados hasta una dimensión objetivo $(10 \mathrm{~mm} * 410 \mathrm{~mm})$ y lijados con grano 120 hasta un espesor objetivo de $13 \mathrm{~mm}$. Los 18 tableros fueron acondicionados en un primer paso a un clima de $20{ }^{\circ} \mathrm{C} / 30 \%$ de humedad relativa hasta que los tableros alcancen su contenido de humedad de equilibrio. La rugosidad de las caras de los tableros MDF fue entonces medida usando un rugosímetro de contacto de principio stylus marca Perthometer (Modelo S4P). La medición comienza con la calibración de los instrumentos. En este experimento la distancia LT de medición fue fijada en $5.6 \mathrm{~mm}$ y la distancia vertical (VB) fue de $250 \mu \mathrm{m}$.

En cada variante fueron realizadas diez mediciones. Durante cada medición el software Perthometer calculó el valor del parámetro $\mathrm{R}_{\mathrm{a}}$ en el último paso del experimento los tableros MDF fueron almacenados a $20{ }^{\circ} \mathrm{C}$ y $85 \%$ de humedad relativa y la rugosidad fue nuevamente evaluada. Todo el experimento tomó nueve semanas; en cada clima los tableros MDF necesitaron tres semanas para alcanzar su humedad de equilibrio. 


\section{RESULTADOS Y DISCUSIÓN}

\section{Influencia de las condiciones climáticas en la rugosidad de tableros de densidad media (MDF) sin recubrir y encolados con resina-MUF}

A los tableros de fibras encolados con resina-MUF se les calculó su humedad de equilibrio después de haber sido almacenados bajo diferentes condiciones climáticas $\left(20^{\circ} \mathrm{C} / 30 \%\right.$ h.r., $20^{\circ} \mathrm{C} / 65 \%$ h.r. y $20^{\circ} \mathrm{C} / 85 \%$ h.r). Como muestra la Figura 1, el contenido de humedad de todos los tableros de fibras con resina-MUF aumenta con el incremento de la humedad relativa independiente del material lignocelulósico usado para la manufactura de los tableros. Tableros de fibras encolados con resinaMUF hechos de fibras frescas y recicladas en sus capas superficiales muestran más ó menos el mismo aumento en el contenido de humedad al incrementarse la humedad relativa durante el almacenado. Para los tableros de fibra con resinas-MUF y hechos con fibras frescas y recicladas el contenido de humedad de equilibrio después el almacenaje a $20^{\circ} \mathrm{C} / 30 \%$ h.r. fue $3.0 \%$ y $2.9 \%$ respectivamente. Después de almacenar los mismos tableros pero ahora en clima $20^{\circ} \mathrm{C} / 65 \%$ h.r. el contenido de humedad de ambos tipos de tableros incremento hasta $7.5 \%$.

El contenido de humedad más alto alcanzado por ambos tableros fue de $(9.5 \%$ y $9.4 \%$ respectivamente) cuando estos fueron climatizados a $20^{\circ} \mathrm{C} / 85 \%$ h.r. El contenido de humedad de equilibrio alcanzado por los tableros de fibra con resina-MUF, con capa central de fibra y capas externas de partículas de corcho, fue menor que aquellos tableros de fibra con resina-MUF donde se uso fibras frescas y recicladas en las capas externas.

El contenido de humedad de equilibrio de tableros de fibra de densidad media MDF con resinasMUF y capas externas de partículas de corcho en la superficie, aumentaron con el incremento de la humedad relativa desde $2.5 \%\left(20^{\circ} \mathrm{C} / 30 \%\right.$ h.r. $)$, a sobre $6.3 \%\left(20^{\circ} \mathrm{C} / 65 \%\right.$ h.r. $)$ hasta llegar a $8.1 \%$ $\left(20^{\circ} \mathrm{C} / 85 \%\right.$ h.r).

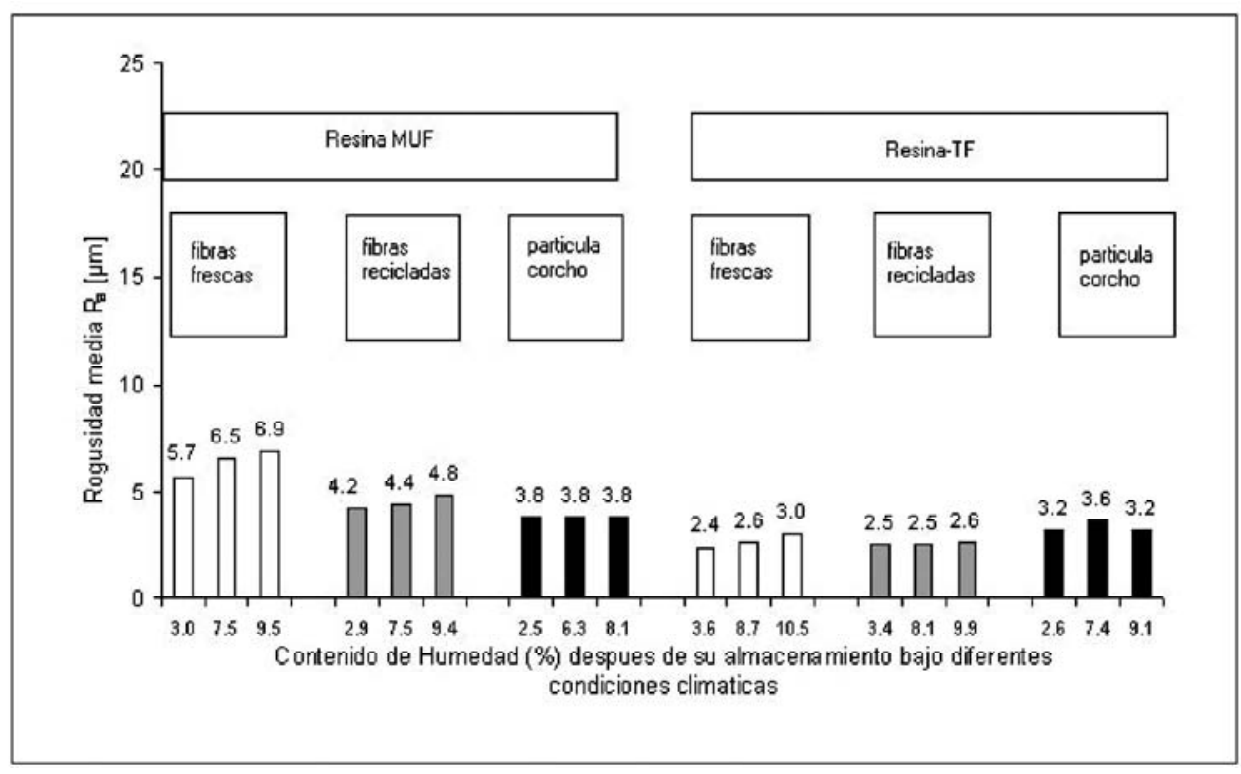

Figura 1: Rugosidad promedio Ra $(\mu \mathrm{m})$ de tableros de fibra de densidad media (MDF), encolados con resinas MUF- y TF hechos usando diferentes materias primas en las capas superficiales (fibras frescas, fibras recicladas y partículas de corcho) después de climatizar bajo diferentes condiciones $\left(20^{\circ} \mathrm{C} / 30 \%\right.$ humedad relativa, $20^{\circ} \mathrm{C} / 65 \%$ humedad relativa y $20^{\circ} \mathrm{C} / 85 \%$ humedad relativa) 
También la Figura 1 muestra la influencia de la materia prima y el contenido de humedad en la rugosidad de tableros de fibra de densidad media MDF con resinas-MUF. Como se puede ver desde la Figura 1, tableros de fibra de densidad media MDF con resinas-MUF y fibras frescas muestran un alta rugosidad independiente del contenido de humedad $\left(\mathrm{R}_{\mathrm{a}}=6.5 \mu \mathrm{m}\right.$ a $\left(20^{\circ} \mathrm{C} / 65 \%\right.$ h.r. $)$ en comparación a MDF con fibra reciclada $\left(\mathrm{R}_{\mathrm{a}}=4.4 \mu \mathrm{m}\right.$ a $20^{\circ} \mathrm{C} / 65 \%$ h.r.). Por otra parte los tableros fibra de densidad media MDF con resinas-MUF pero con corcho en las capas externas tienen superficies mas suaves $\left(\mathrm{Ra}=3.8 \mu \mathrm{m}\right.$ a $20^{\circ} \mathrm{C} / 65 \%$ h.r.). Sin embargo, la Figura 1 muestra la influencia general de diferentes condiciones climáticas en la rugosidad de tableros de fibra de densidad media MDF con resinas-MUF.

La rugosidad de tableros de fibra de densidad media MDF con resinas-MUF con fibras frescas y recicladas incrementa con el aumento del contenido de humedad. El valor de $\mathrm{R}_{\mathrm{a}}$ de tableros de fibra de densidad media MDF con resinas-MUF con fibras frescas incrementa con el alza de la humedad relativa en la región de $30 \%$ h.r sobre $65 \%$ h.r. hasta $85 \%$ h.r. desde $5.7 \mu \mathrm{m}$ sobre $6.5 \mu \mathrm{m}$ hasta $6.9 \mu \mathrm{m}$. En el caso de fibras recicladas, $\mathrm{R}_{\mathrm{a}}$ se incrementa desde $4.2 \mu \mathrm{m}$ hasta $4.8 \mu \mathrm{m}$. Sin embargo los resultados indican que tableros de fibra de densidad media MDF con resinas-MUF con partículas de corcho en su cara superficial tienen un comportamiento diferente, no hay un incremento detectable en la rugosidad de los tableros observado debido a un incremento en el contenido de humedad de los tableros.

\section{Influencia de las condiciones climáticas en la rugosidad de tableros de densidad media (MDF) sin recubrir y encolados con resina-TF}

Los tableros de densidad media (MDF) sin recubrir y encolados con resina-TF fueron almacenados bajo diferentes condiciones climáticas $\left(20^{\circ} \mathrm{C} / 30 \%\right.$ h.r., $20^{\circ} \mathrm{C} / 65 \%$ h.r. y $20^{\circ} \mathrm{C} / 85 \%$ h.r $)$, para obtener posteriormente su humedad de equilibrio en cada uno de ellos. Lo anterior se puede ver en la Figura 1 donde el contenido de humedad de todos los tableros de fibra (MDF) sin recubrir y encolados con resina-TF se incrementa con humedades relativas altas independiente del material lignocelulósico utilizado para su manufactura. Tableros (MDF) hechos con fibra frescas en su superficie y encolados con resina-TF muestran en comparación a (MDF) hechos con fibra reciclada un contenido de humedad ligeramente más alto con el incremento de la humedad relativa durante el almacenaje. El contenido de humedad de tableros de fibras de densidad media (MDF) sin recubrir y encolados con resina-TF hechos con partículas de corcho en la superficie son más altos en general si se comparan con aquellos tableros donde se usó fibras frescas y recicladas. El contendido de humedad de equilibrio de tableros de fibras de densidad media (MDF) sin recubrir y encolados con resina-TF hechos con partículas de corcho en la superficie aumenta con el climatizado comenzando desde un $2.6 \%$ de equilibrio $\left(20^{\circ} \mathrm{C} / 30 \%\right.$ h.r. $)$ sobre $7.4 \%\left(20^{\circ} \mathrm{C} / 65 \%\right.$ h.r. $)$ hasta $9.1 \%$ a $\left(20^{\circ} \mathrm{C} / 85 \%\right.$ h.r. $)$.

También la Figura 1 muestra la influencia de la materia prima en la rugosidad de tableros MDF encolados con resina-TF. Estos tableros cuando se ocupa fibras frescas y recicladas en su manufactura presentaron un nivel homogéneo de rugosidad en sus superficies. Por otro lado los tableros MDF encolados con resina-TF pero con partículas de corcho en sus capas superficiales presentaron altas rugosidades pero también homogéneas. En la Figura 1 se ve como cambios en las condiciones climáticas afectan ligeramente la rugosidad de los tableros MDF encolados con resina-TF. Solo los tableros MDF encolados con resina-TF donde se usó fibras frescas muestran un incremento ligero en la rugosidad debido a un incremento en el contenido de humedad de los tableros.

Finalmente, independiente de la materia prima usada para hacer tableros de fibra, un incremento en el contenido de humedad de equilibrio de los tableros fue visualizado a medida que la humedad relativa incrementaba desde $30 \%$ h.r. sobre $65 \%$ h.r hasta $85 \%$ h.r. Sin embargo la influencia de los adhesivos usados en la humedad de equilibrio de los tableros manufacturados es obvia. Tableros MDF encolados con resina-TF obtienen en general contenidos de humedad de equilibrio más altos en comparación con tableros MDF encolados con resina-MUF. Esto nuevamente puede ser debido a la naturaleza alcalina de la resina-TF. 
Tableros MDF encolados con resina-TF poseen en general un contenido de humedad de equilibrio mayor si los comparamos con tableros de MDF encolados con resina- MUF. En general, tableros MDF encolados con resina-TF tienen superficies más suaves que los tableros encolados con resina- MUF independiente de la materia prima utilizada. Esto últimos tableros incrementan su rugosidad con altos contenidos de humedad en los tableros, sin embargo de tableros con resina-TF solo un pequeño incremento en la rugosidad fue observado debido al incremento en la humedad de equilibrio. Una comparación entre materias primas indica que el uso de fibras frescas produce superficies más rugosas que si se usan fibras recicladas. Por otra parte tableros MDF con partículas de corcho en las capas superficiales y encoladas con resinas-TF y MUF, no muestran grandes cambios en su rugosidad cuando el contenido de humedad de los tableros aumenta.

\section{Análisis estadístico de los resultados}

Dentro del marco de este estudio el grado de interacción entre factores (variables independientes) como: Materia prima (fibras frescas, fibras recicladas, partículas de corcho); Adhesivo MUF (melaminaurea-formaldehído) y TF (tanino-formaldehído); condiciones climáticas $\left(20^{\circ} \mathrm{C} / 30 \%\right.$ h.r., $20^{\circ} \mathrm{C} / 65 \%$ h.r. y $20^{\circ} \mathrm{C} / 85 \%$ h.r. y las respuestas (variables dependientes) de los tableros de fibras desnudos como: rugosidad media $\left(\mathrm{R}_{\mathrm{a}}\right)$ y contendido de humedad (\%) fue determinada. Los datos obtenidos fueron estadísticamente analizados con dos pruebas estadísticas (análisis de Anova y Tuckey). La Tabla 2 ayuda a entender como los datos para el análisis de Anova fueron preparados.

Tabla 2. Matriz para el Análisis Anova, factores (variables independientes) y respuestas (variables dependientes) de los experimentos.

\begin{tabular}{|c|c|c|c|c|}
\hline \multicolumn{3}{|c|}{$\begin{array}{c}\text { Factores } \\
\text { (variables independientes) }\end{array}$} & \multicolumn{2}{|c|}{$\begin{array}{c}\text { Respuestas } \\
\text { (variables dependientes) }\end{array}$} \\
\hline \multirow[t]{2}{*}{$\begin{array}{l}\text { Materia } \\
\text { Prima }\end{array}$} & \multirow[t]{2}{*}{$\begin{array}{l}\text { Tipo de } \\
\text { resina }\end{array}$} & $\begin{array}{l}\text { Condiciones } \\
\text { Climaticas }\end{array}$ & $\begin{array}{c}\text { Rugosidad media } \\
\left(\mathrm{R}_{a}\right)\end{array}$ & $\begin{array}{l}\text { Contenido de } \\
\text { humedad }\end{array}$ \\
\hline & & ${ }^{\circ} \mathrm{C} /$ hum. rel. \% & $\mu \mathrm{m}$ & $\%$ \\
\hline \multirow{6}{*}{$\begin{array}{l}\text { Fibras } \\
\text { Frescas }\end{array}$} & \multirow{3}{*}{$\begin{array}{l}\text { Resina- } \\
\text { MUF }\end{array}$} & $20 / 30$ & 5.7 & 3.0 \\
\hline & & $20 / 65$ & 6.5 & 7.5 \\
\hline & & $20 / 85$ & 6.9 & 9.5 \\
\hline & \multirow{3}{*}{ Resina-TF } & $20 / 30$ & 2.4 & 3.6 \\
\hline & & $20 / 65$ & 2.6 & 8.7 \\
\hline & & $20 / 85$ & 3.0 & 10.5 \\
\hline \multirow{6}{*}{$\begin{array}{l}\text { Recycled } \\
\text { fibers }\end{array}$} & \multirow{3}{*}{$\begin{array}{l}\text { Resina- } \\
\text { MUF }\end{array}$} & $20 / 30$ & 4.2 & 2.9 \\
\hline & & $20 / 65$ & 4.4 & 7.5 \\
\hline & & $20 / 85$ & 4.8 & 9.4 \\
\hline & \multirow{3}{*}{ Resina-TF } & $20 / 30$ & 2.5 & 3.4 \\
\hline & & $20 / 65$ & 2.5 & 8.1 \\
\hline & & $20 / 85$ & 2.6 & 9.9 \\
\hline \multirow{6}{*}{$\begin{array}{c}\text { Cork } \\
\text { particles }\end{array}$} & \multirow{3}{*}{$\begin{array}{l}\text { Resina- } \\
\text { MUF }\end{array}$} & $20 / 30$ & 3.8 & 2.5 \\
\hline & & $20 / 65$ & 3.8 & 6.3 \\
\hline & & $20 / 85$ & 3.8 & 8.1 \\
\hline & & $20 / 30$ & 3.2 & 2.6 \\
\hline & \multirow[t]{2}{*}{ Resina-TF } & $20 / 65$ & 3.6 & 7.4 \\
\hline & & $20 / 85$ & 3.2 & 9.1 \\
\hline
\end{tabular}


La Tabla 3 muestra los resultados del análisis de Anova. Esta prueba evalúa los efectos de los factores sobre las respuestas en dos niveles de significancia, $0.05 \%$ de probabilidad estadística como significante y $0.01 \%$ de probabilidad estadística como altamente significante. Lo anterior puede ser visualizado desde la tabla 3, que factor por si mismo o en interacción con otro factor determina diferencias significativas en los valores de las respuestas (rugosidad y contenido de humedad).

Tabla 3: Resultados del análisis de Anova. Grado de significancia expresado como probabilidad bajo dos niveles $(0.05 \%$ de probabilidad como significativo y $0.01 \%$ de probabilidad como altamente significativo).

\begin{tabular}{|l|c|c|c|c|c|c|}
\hline & \multicolumn{3}{|c|}{$\begin{array}{c}\text { Factores } \\
\text { (variables independientes) }\end{array}$} & \multicolumn{2}{c|}{$\begin{array}{c}\text { Interrelación entre factores } \\
\text { (variables independientes) }\end{array}$} \\
\hline $\begin{array}{l}\text { Respuestas } \\
\text { (variables } \\
\text { dependientes) }\end{array}$ & Materia prima & Resina & Climatizado & $\begin{array}{c}\text { Materia prima / } \\
\text { Resina }\end{array}$ & $\begin{array}{c}\text { Materia prima / } \\
\text { Climatizado }\end{array}$ & $\begin{array}{c}\text { Resina / } \\
\text { Climatizado }\end{array}$ \\
\hline $\begin{array}{l}\text { Rugosidad } \\
\text { promedio } \\
\left(\mathrm{R}_{\mathrm{a}}\right) \mu \mathrm{m}\end{array}$ & $<0.0001$ & 0.0008 & 0.0257 & 0.0002 & no significante & no significante \\
\hline $\begin{array}{l}\text { Humedad de } \\
\text { equilibrio } \\
(\%)\end{array}$ & 0.0011 & 0.0010 & $<0.0001$ & no significante & no significante & no significante \\
\hline
\end{tabular}

\section{Rugosidad promedio $\left(R_{a}\right)$}

De acuerdo con la tabla 3 el material lignocelulósico $(<0.0001)$ es el factor más importante que afecta la rugosidad de tableros MDF encolados con resinas MUF y TF. La prueba también indica una significante interacción entre la materia prima y las resinas. Esta interacción fue analizada mediante Tuckey. Una comparación para cada resina fue hecha. Los resultados muestran que la rugosidad de tableros de fibra encolados con resina-MUF hechos con fibras frescas es significativamente diferente en comparación a los tableros encolados con resina-MUF pero hechos con fibras recicladas y partículas de corcho en la superficie. La rugosidad de tableros de fibras encolados con resina-TF con capas superficiales hechas de partículas de corcho es significativamente diferente si la comparamos a la encontrada en tableros de fibra encolados con resina-TF pero con capas superficiales hechas de fibras frescas y recicladas.

\section{Contenido de humedad (\%)}

Sin duda esta variable respuesta es signicativamente controlada por el factor de condición climática, sin embargo, las resinas y la materia prima pueden también influir en el contenido de humedad significativamente. El análisis de Tuckey muestra que tanto las resinas como las materias primas influyen en el contenido de humedad de los tableros, el cual finalmente determina cambios en los niveles de rugosidad alcanzados. 


\section{CONCLUSIONES}

- Las condiciones climáticas son el factor que domina el contenido de humedad de equilibrio en los tableros, sin embargo, tanto las resinas como las materias primas utilizadas para su manufactura influyen en él.

- El contenido de humedad de equilibrio de los tableros modifica la rugosidad.

- El tipo de resina influye en la rugosidad, en general tableros de fibra de densidad media MDF encolados con resina-TF tiene superficies más suaves que los tableros MDF encolados con resinaMUF independiente de la materia prima usada, condición particularmente importante al aplicar recubrimientos decorativos.

- El material lignocelulósico es el factor más importante que afecta la rugosidad de los tableros MDF encolados con resinas-MUF y TF.

\section{BIBLIOGRAFÍA}

Hiziroglu, S. 1996. Surface roughness analysis of wood composites: A stylus method. Forest Products Journal 46 (7/8): 67-72

Östman, B. 1983. Surface roughness of wood based panels after aging. Forest Products Journal 33 (7): $35-42$

PDI. 1998. Surface metrology guide-home. Precision Divices Inc. http://www.predev.com

Roffael, E. 1993. Formaldehyde release from particleboard and other wood based panels. Malayan Forest Records No.37. ISBN: 983-9592-15-7

Roffael, E.; Schäfer, M. 1997. Die Rohstofffrage in Holz und Kunststoffvearbeitung. Recyceltes Material minimiert Kosten und entlastet die Umwelt. Holz- und Kunststoffverabeitung 36 (4): 36 - 40 